\title{
Capacidade funcional do idoso com demência de Alzheimer: $O$ papel da musicoterapia
}

\section{Functional ability of the elderly with Alzheimer's disease: The role of music therapy}

\author{
Gabriella Katherine Lopes*,** \\ Helena Camargo Porto* \\ Isabella Veroneze Spera* \\ Jamile Silva Marques* \\ Juliana Domingues Brandão* \\ Leonardo Carvalho de Souza* \\ Maria Elisa Gonzalez Manso***
}

\begin{abstract}
Resumo: Este estudo trata-se de uma revisão integrativa da literatura que analisou seis artigos que circundam as faculdades da musicoterapia e o efeito desta sobre os sintomas da demência de Alzheimer (DA) em idosos. A musicoterapia é um tratamento não farmacológico utilizado nas intervenções de diversas doenças, como, na DA. Essa terapia demonstrou benefícios quanto a redução de sintomas relacionados a patologia em questão, como por exemplo, alterações positivas da ansiedade, humor e agitação.
\end{abstract}

Palavras-chaves: Musicoterapia. Alzheimer.

\begin{abstract}
This study is an integrative literature review that analyzed six articles surrounding the faculties of music therapy and its effect on the symptoms of Alzheimer's dementia (AD) in the elderly. Music therapy is a non-pharmacological treatment used in interventions for various diseases, such as in AD. This therapy has shown benefits in reducing symptoms related to the condition in question, such as positive changes in anxiety, mood and agitation.
\end{abstract}

Keywords: Musict Terapy. Alzheimer's.

\footnotetext{
*Discente da Faculdade de Medicina do Centro universitário São Camilo

** E-mail: gabriella_lopeskath@live.com

***Docente da Faculdade de Medicina do Centro universitário São Camilo
} 


\section{Introdução}

O número de idosos no Brasil cresce $18 \%$ a cada 5 anos e ultrapassou 30 milhões em 2017. Além disso, estudos demonstram que a prevalência de demências dobra a cada 5 anos a partir dos 60 anos de idade e em 2050 um em cada três brasileiros serão idosos ${ }^{1}$. Estes dados ressaltam a importância de investimentos nas práticas de saúde que incluem o auxílio no tratamento dos déficits cognitivos, como por exemplo, a musicoterapia na DA.

A doença em questão caracteriza-se inicialmente pelo ligeiro declínio das capacidades intelectuais, como prejuízo da memória recente, dificuldade de manter a atenção, de associação e orientação, sobre tudo espacial, seguido de lentidão do pensamento e da capacidade de executar tarefas sociais e econômicas ${ }^{2}$.

Os recursos terapêuticos das práticas farmacológicas para portadores da doença de Alzheimer são bastante discutidos nos artigos acadêmicos, no entanto, são pouco abordados os tratamentos não medicamentosos, que, segundo estudos, aliados ao processo medicamentoso, podem ser capazes de produzir resultados positivos ${ }^{3}$.

Dentre as terapias que auxiliam o tratamento farmacológico do doente com a DA, de acordo com a literatura, a musicoterapia pode ser definida como uma terapia auto expressiva, que "estimula o potencial criativo e a ampliação da capacidade comunicativa, mobilizando aspectos biológicos, psicológicos e culturais do indivíduo" ${ }^{4}$. Para esse fim, a música é principal ferramenta de trabalho.

Atualmente estudos demonstram a importância de tratamentos musicoterapêuticos no âmbito da saúde mental. Através da análise de relatórios é possível observar a singularidade de cada paciente com DA, e esta é expressa nas sessões de musicoterapia, individuais ou em grupo 4 .

O objetivo deste estudo, portanto, é realizar uma revisão integrativa da literatura acerca do papel da musicoterapia como tratamento alternativo no manejo do paciente com demência de Alzheimer. 


\section{Metodologia}

A obtenção dos artigos ocorreu pela internet em setembro de 2018, através da consulta à MEDLINE, LILACS, IBECS e BRISA/RedTESA. Os dados foram coletados utilizando as palavras-chaves, encontradas no DeCS (Descritores em Ciências da Saúde): demência de Alzheimer e musicoterapia. Foram encontrados 139 artigos relacionados aos descritores, dentre esses, 6 foram selecionados pelas temáticas de exclusão como, texto completo, ano de publicação (2015-2018) e exclusão por título e repetição, como demonstrado no fluxograma abaixo.

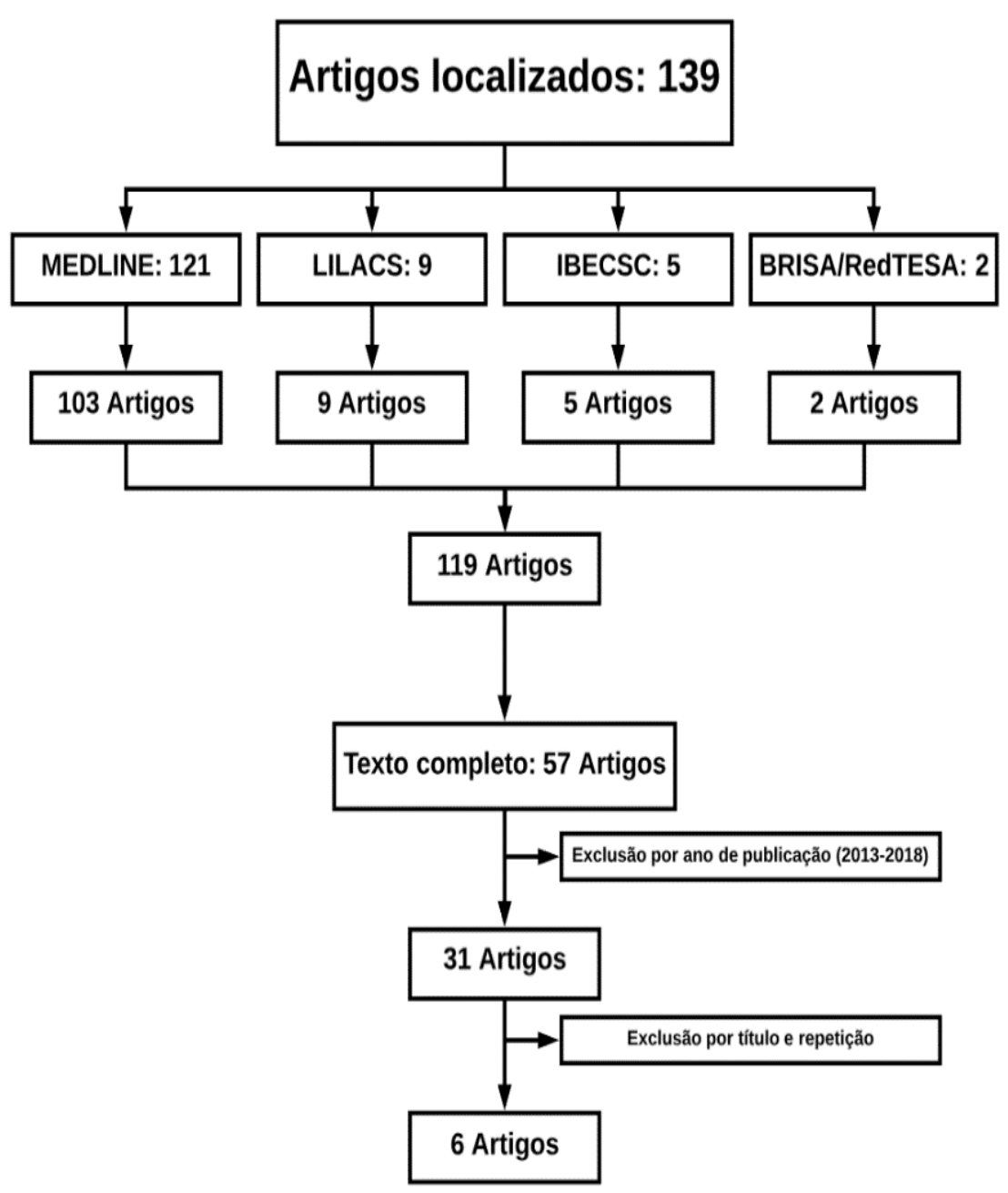


Com base nos estudos analisados foram observados os seguintes resultados:

Quadro 1 - Resultados dos estudos mencionados neste trabalho

\begin{tabular}{|c|c|c|c|c|}
\hline Autor & Título & Metodologia & Conclusão & Revista \\
\hline $\begin{array}{l}\text { ALEIXO } \\
\text { MAR, } \\
\text { SANTOS RL, } \\
\text { DOURADO } \\
\text { MCN. }\end{array}$ & $\begin{array}{l}\text { Eficácia da } \\
\text { musicoterapia nos } \\
\text { sintomas } \\
\text { neuropsiquiátricos } \\
\text { da demência: } \\
\text { revisão sistemática }\end{array}$ & $\begin{array}{l}\text { Artigo de } \\
\text { revisão } \\
\text { sistemática }\end{array}$ & $\begin{array}{l}\text { Embora haja relatos de } \\
\text { eficácia da musicoterapia } \\
\text { na diminuição dos } \\
\text { sintomas } \\
\text { neuropsiquiátricos da } \\
\text { demência, a área ainda } \\
\text { necessita de estudos } \\
\text { randomizados que visem à } \\
\text { solução de problemas } \\
\text { metodológicos } \\
\text { importantes, como a } \\
\text { falta de abordagens } \\
\text { padronizadas }{ }^{5} \text {. }\end{array}$ & $\begin{array}{l}\text { J Bras } \\
\text { Psiquiatr. } \\
2017 ; 66(1): 5 \\
2-61\end{array}$ \\
\hline $\begin{array}{l}\text { GÓMEZ- } \\
\text { ROMERO M, } \\
\text { JIMÉNEZ- } \\
\text { PALOMARES } \\
\text { M, } \\
\text { RODRÍGUEZ- } \\
\text { MANSILLA J, } \\
\text { FLORES- } \\
\text { NIETO A, } \\
\text { GARRIDO- } \\
\text { ARDILA EM, } \\
\text { GONZÁLEZ- } \\
\text { LÓPEZ-ARZA } \\
\text { MV. }\end{array}$ & $\begin{array}{l}\text { Benefits of music } \\
\text { therapy on } \\
\text { behaviour disorders } \\
\text { in subjects } \\
\text { diagnosed with } \\
\text { dementia: A } \\
\text { systematic review }\end{array}$ & $\begin{array}{l}\text { Artigo de } \\
\text { revisão } \\
\text { sistemática }\end{array}$ & $\begin{array}{l}\text { Foi concluído, do artigo } \\
\text { lido, que existem } \\
\text { evidências que } \\
\text { demonstram a melhoria na } \\
\text { ansiedade e agitação dos } \\
\text { pacientes com demência } \\
\text { devido à musicoterapia, } \\
\text { entretanto, o número de } \\
\text { pacientes para análise é } \\
\text { limitado e há baixo } \\
\text { número de estudos a } \\
\text { respeito do assunto }{ }^{6} \text {. }\end{array}$ & $\begin{array}{l}\text { Elsevier } \\
\text { España, } \\
\text { S.L.U., } 2017\end{array}$ \\
\hline $\begin{array}{l}\text { GALLEGO } \\
\text { MG, GARCÍA } \\
\text { JG. }\end{array}$ & $\begin{array}{l}\text { Music therapy and } \\
\text { Alzheimer's } \\
\text { disease: Cognitive, } \\
\text { psychological, and } \\
\text { behavioural effects }\end{array}$ & $\begin{array}{l}\text { Pesquisa } \\
\text { cientifica }\end{array}$ & $\begin{array}{l}\text { A musicoterapia é uma } \\
\text { intervenção barata e } \\
\text { agradável, sem efeitos } \\
\text { adversos e surgiu como } \\
\text { uma alternativa promissora } \\
\text { para pacientes com } \\
\text { demência, uma vez que } \\
\text { melhora o humor e reduz } \\
\text { os problemas de } \\
\text { comportamento } \\
\text { desencadeados por } \\
\text { condições estressantes }{ }^{7} \text {. }\end{array}$ & $\begin{array}{l}\text { Elsevier } \\
\text { España, } \\
\text { S.L.U., } 2017\end{array}$ \\
\hline
\end{tabular}




\begin{tabular}{|c|c|c|c|c|}
\hline $\begin{array}{l}\text { OLIVEIRA } \\
\text { AT, ROSA } \\
\text { AAS, BRAUN } \\
\text { AM, MICCO } \\
\text { DK, ERTHAL } \\
\text { IN, PECOITS } \\
\text { RV. }\end{array}$ & $\begin{array}{lr}\text { A música } & \text { no } \\
\text { controle } & \text { de } \\
\text { sintomas } & \\
\text { relacionados à } & \text { à } \\
\text { demência em idosos }\end{array}$ & $\begin{array}{l}\text { Artigo de } \\
\text { Revisão } \\
\text { Sistemática }\end{array}$ & $\begin{array}{l}\text { Os } \\
\text { neuropsiquiátricos } \\
\text { pareceram ser os mais } \\
\text { afetados positivamente } \\
\text { pela terapia musical e as } \\
\text { relações interpessoais e a } \\
\text { linguagem apresentaram } \\
\text { grande evolução. Por outro } \\
\text { lado, não houveram } \\
\text { melhoras } \\
\text { relevantes }{ }^{8} \text {. cognitivas }\end{array}$ & $\begin{array}{l}\text { ACTA } \\
\text { MÉDICA - } \\
\text { LIGAS } \\
\text { ACADÊMIC } \\
\text { AS } \\
\text { Vol. 39, n. } 1 \\
(2018)\end{array}$ \\
\hline $\begin{array}{l}\text { MILLÁN- } \\
\text { CALENTI JC, } \\
\text { LORENZO- } \\
\text { LÓPEZ L, } \\
\text { ALONSO- } \\
\text { BÚA B, } \\
\text { LABRA C, } \\
\text { GONZÁLEZ- } \\
\text { ABRALDES I, } \\
\text { MASEDA A. }\end{array}$ & $\begin{array}{l}\text { Optimal non } \\
\text { pharmacological } \\
\text { management of } \\
\text { agitation in } \\
\text { Alzheimer's } \\
\text { disease: challenges } \\
\text { and solutions } \\
\text { 3: Artigo de revisão } \\
\text { sistemática }\end{array}$ & $\begin{array}{l}\text { Artigo de } \\
\text { revisão } \\
\text { sistemática }\end{array}$ & $\begin{array}{l}\text { Esta revisão constatou que } \\
\text { a musicoterapia é uma } \\
\text { intervenção não- } \\
\text { farmacológica eficaz para } \\
\text { reduzir a agitação em } \\
\text { pacientes com Doença de } \\
\text { Alzheimer } \\
\text { institucionalizada, } \\
\text { particularmente quando a } \\
\text { intervenção implica } \\
\text { música individualizada e } \\
\text { interativa. No entanto, } \\
\text { mais evidências sobre os } \\
\text { efeitos a longo prazo dessa } \\
\text { terapia são necessárias }{ }^{3} \text {. }\end{array}$ & $\begin{array}{l}\text { Dove } \\
\text { Medical } \\
\text { Press, } 22 \\
\text { February } \\
\text { 2016 Volume } \\
\text { 2016:11 } \\
\text { Pages 175- } \\
\text { 184 }\end{array}$ \\
\hline $\begin{array}{l}\text { RAGLIO A, } \\
\text { FILIPPI S, } \\
\text { BELLANDI } \\
\text { D, } \\
\text { STRAMBA- } \\
\text { BADIALE M. }\end{array}$ & $\begin{array}{l}\text { Global music } \\
\text { approach to } \\
\text { persons with } \\
\text { dementia: evidence } \\
\text { and practice }\end{array}$ & $\begin{array}{l}\text { Artigo de } \\
\text { revisão } \\
\text { sistemática }\end{array}$ & $\begin{array}{l}\text { O modelo GMA-D } \\
\text { (Abordagem global } \\
\text { musical para pessoas com } \\
\text { demência) deve ser } \\
\text { considerado uma } \\
\text { intervenção não } \\
\text { farmacológica de baixo } \\
\text { custo e um método } \\
\text { terapêutico-reabilitador } \\
\text { para a redução dos } \\
\text { sintomas comportamentais } \\
\text { e psicológicos da } \\
\text { demência, para estimular } \\
\text { as funções } \\
\text { cognitivas e para aumentar } \\
\text { a qualidade de vida geral } \\
\text { da PCD (pacientes com } \\
\text { demência) }{ }^{9} \text {. }\end{array}$ & $\begin{array}{l}\text { Clinical } \\
\text { Interventions } \\
\text { in Aging. } 6 \\
\text { October } 2014\end{array}$ \\
\hline
\end{tabular}




\section{Discussão}

As terapias não farmacológicas tornam-se cada vez mais populares na gestão dos sintomas comportamentais comuns nos estágios intermediários ou posteriores da demência, como a euforia. O uso da musicoterapia auxilia no manejo da agitação em pacientes institucionalizados com Demência de Alzheimer, particularmente quando a intervenção inclui música individualizada e familiar, relacionado a lembranças de experiências pessoais; realizada de forma interativa, incluindo palmas, cantos e danças ${ }^{7}$.

Com base nos estudos analisados para a realização desta revisão integrativa da literatura, foi evidenciado que, apesar de existirem análises comprovadas cientificamente sobre a ação da música nos sistemas límbico e paralímbico do cérebro ${ }^{9}$, não há metodologias padronizadas para avaliar a eficácia da musicoterapia no desenvolvimento da capacidade funcional do idoso com demência de Alzheimer. Isto dificulta a aplicação desta terapia de maneira eficaz, já que, a execução desta é feita de maneira difusa e muitas vezes imprecisa ${ }^{7}$.

Além disso, a música estimula, principalmente, a função motora e intelectual e, no aspecto psicológico, facilita a comunicação e a relação entre as emoções e expressões, assim como, a fala e funções cognitivas relacionadas a memória e atenção. Ademais, funcionários que conviveram com idosos submetidos a musicoterapia relataram perceber alterações positivas em relação ao comportamento desses pacientes durante as sessões, tais como: aumento de contato visual, expressões faciais e alterações positivas de humor que remetem a uma melhoria nas relações interpessoais ${ }^{8}$.

A musicoterapia, com o intermédio de um profissional qualificado para realizar e orientar, possui várias formas de aplicação. Como por exemplo, intervenções musicais, cuidadores cantando, música ambiente. Além disso, a musicoterapia pode ser realizada de forma ativa, que apresenta duas abordagens, fundamentos psicológicos e reabilitativos, contando, também, com participação da família do paciente com DA ${ }^{7}$. 


\section{Conclusão}

Com base nos estudos revisados, foi concluído que a musicoterapia é relevante como um tratamento de baixo custo, que altera positivamente o humor, agitação e ansiedade, melhorando as relações interpessoais dos pacientes com sintomas de DA praticantes dessa terapia. No entanto, as pesquisas realizadas sobre os efeitos da musicoterapia em pacientes com sintomas de DA são poucos, o que dificulta sua aplicação de maneira efetiva. Dessa forma, a padronização dos estudos sobre a temática se torna necessária para melhor avaliar os benefícios e a aplicação desta forma terapêutica não medicamentosa no tratamento e no acompanhamento da progressão sintomática da DA. 


\section{Referências}

1. LEAL, LN. As regras têm como objetivo proteger o investimento feito pelo Estadão na qualidade constante de seu jornalismo. Estadão, São Paulo, 29 de agosto de 2016. Disponível em: https://brasil.estadao.com.br/noticias/geral,populacao-idosa-vai-triplicar-entre-2010-e2050-aponta-publicacao-do-ibge,10000072724. Acesso em: 13/09/2019.

2. CONVERSO ME, IARTELLI I. Caracterização e análise do estado mental e funcional de idosos institucionalizados em instituições públicas de longa permanência. J Bras Psiquiatr. 2007;56(4): 267-272.

3. MiLLÁN-CALENTI JC, LORENZO-LÓPEZ L, ALONSO-BÚA B, LABRA C, GONZÁLEZ-ABRALDES I, MASEDA A. Optimal nonpharmacological management of agitation in Alzheimer's disease: challenges and solutions. Dovepress. 2018;177.33.19.3.

4. REIS MRR, FREIRE MH. A canção de apresentação como um recurso de musicoterapia na saúde mental. Incantare. 2018;2317-417X.

5. ALEIXO MAR, SANTOS RL, DOURADO MCN. Efficacy of music therapy in the neuropsychiatric symptoms of dementia: systematic review. J Bras Psiquiatr. 2017;66(1):5261.

6. GÓMEZ-ROMERO M, JIMÉNEZ-PALOMARES M, RODRÍGUEZ-MANSILLA J, FLORES-NIETO A, GARRIDO-ARDILA EM, GONZÁLEZ-LÓPEZ-ARZA MV.

Beneficios de la musicoterapia en las alteraciones conductuales de la demencia. Neurología. 2017;32:253-263.

7. GALLEGO MG, GARCÍA JG. Music therapy and Alzheimer's disease: Cognitive, psychological, and behavioural effects. Neurología. 2017;32:300-308.

8. OLIVEIRA AT, ROSA AAS, BRAUN AM, MICCO DK, ERTHAL IN, PECOITS RV. A música no controle de sintomas relacionados à demência em idosos. ACTA MEDICA PUCRS. 2018;0103-5037. 
9. RAGLIO A, FILIPPI S, BELLANDI D, STRAMBA-BADIALE M. Global music approach to persons with dementia: evidence and practice. Dovepress. 2014;10.2147/CIA.S71388. 